\title{
Nonadiabatic Spin Torque Investigated Using Thermally Activated Magnetic Domain Wall Dynamics
}

\author{
M. Eltschka, M. Wötzel, ${ }^{*}$ J. Rhensius, ${ }^{\dagger}$ S. Krzyk, U. Nowak, and M. Kläui ${ }^{\ddagger}$ \\ Fachbereich Physik, Universität Konstanz, Universitätsstraße 10, 78457 Konstanz, Germany \\ T. Kasama and R. E. Dunin-Borkowski \\ Center for Electron Nanoscopy, Technical University of Denmark, 2800 Kongens Lyngby, Denmark \\ L. J. Heyderman \\ Laboratory for Micro- and Nanotechnology, Paul Scherrer Institut, 5232 Villigen PSI, Switzerland
}

H. J. van Driel and R. A. Duine

Institute for Theoretical Physics, Utrecht University, Leuvenlaan 4, 3584 CE Utrecht, The Netherlands (Received 23 February 2010; published 29 July 2010)

\begin{abstract}
Using transmission electron microscopy, we investigate the thermally activated motion of domain walls (DWs) between two positions in Permalloy $\left(\mathrm{Ni}_{80} \mathrm{Fe}_{20}\right)$ nanowires at room temperature. We show that this purely thermal motion is well described by an Arrhenius law, allowing for a description of the DW as a quasiparticle in a one-dimensional potential landscape. By injecting small currents, the potential is modified, allowing for the determination of the nonadiabatic spin torque: $\beta_{t}=0.010 \pm 0.004$ for a transverse DW and $\beta_{v}=0.073 \pm 0.026$ for a vortex DW. The larger value is attributed to the higher magnetization gradients present.
\end{abstract}

The controlled motion of magnetic domain walls (DWs) by the injection of spin-polarized currents $[1,2]$ has become an exciting field of research, since the detailed understanding of the interplay between the local magnetization and the spin angular momentum of the electron current is of scientific interest and essential for proposed applications [3]. Thermal effects, resulting from Joule heating due to the high injected current densities necessary for DW displacement, have often been neglected and have in general been considered as detrimental in the past. However, when properly analyzed, thermally activated processes at temperatures even well below the Curie temperature can be used to provide a better understanding of the underlying physical mechanisms [4]. Current-induced DW displacement has been demonstrated successfully in experiments [1-3], but the underlying theory describing the torque exerted by spin-polarized conduction electrons on the local magnetization and, in particular, their quantitative values are still subject to controversial discussions [5-8].

In order to account for the effect of the current, two spin torque terms have been added to the Landau-Lifshitz or Gilbert equation, the latter of which reads for the current flowing along the $x$ axis $[5,6,9]$

$$
\begin{aligned}
\frac{\partial \mathbf{S}_{i}}{\partial t}= & -\frac{\gamma}{\mu_{s}} \mathbf{S}_{i} \times \mathbf{H}_{i}(t)+\alpha \mathbf{S}_{i} \times \frac{\partial \mathbf{S}_{i}}{\partial t}-u_{x} \frac{\partial \mathbf{S}_{i}}{\partial x} \\
& +\beta u_{x} \mathbf{S}_{i} \times \frac{\partial \mathbf{S}_{i}}{\partial x},
\end{aligned}
$$

where $\mathbf{H}_{i}(t)$ are the effective fields, $\gamma=g \mu_{B} / \hbar$ is the gyromagnetic ratio, $\mathbf{S}_{i}=\boldsymbol{\mu}_{i} / \mu_{s}$ represents the magnetic moment of unit length with $\mu_{s}=\left|\boldsymbol{\mu}_{i}\right|, u_{x}=j P g \mu_{B} \mid$ $\left(2 e M_{s}\right)$ is an effective velocity for current density $j, P$ is the polarization, and $M_{s}$ is the saturation magnetization [9]. Gilbert damping is described by the constant $\alpha$ and the nonadiabaticity is characterized by the parameter $\beta$. While the third term (adiabatic spin torque) is well understood, the physical contributions to the nonadiabatic torque are still the subject of scientific debate, and both spin-flip scattering and linear momentum transfer due to nonadiabatic transport have been discussed [6-8]. In particular, this torque becomes an effective force in a onedimensional (1D) model [7]. While the purely adiabatic contribution is expected to prevail for wide DW spin structures, the nonadiabatic term has been proposed to be more effective in narrow DWs or vortices where high magnetization gradients occur [8]. However, the relative magnitude of the nonadiabatic term and its relation to the damping constant and to the characteristic transport length scale, which the DW width has to be compared to, are still debated and depend on the model used [7,8,10-12].

Different methods to determine $\beta$ have been put forward with partly contradicting results. One problem has been the influence of thermal effects, which stem from the Joule heating due to the high current densities that are required for DW movement and which can affect the physical mechanisms involved [13]. However, a first step to analyzing the spin torque effect using thermal effects was recently taken, when the thermally activated depinning processes induced by external fields, with simultaneous current injection, were studied in hard magnetic materials 
[14]. DWs can be pinned at artificially structured notches in wires, defects intrinsic to the material or defects caused by fabrication, all of which can generate attractive potential wells $[2,14-17]$. Because of the strong pinning sites in hard magnetic materials [14], DWs can only be depinned by additional external magnetic fields deforming DWs prior to depinning. The resulting complicated spin structures make reliable conclusions about spin torque difficult without direct imaging, which has not been carried out so far.

Thus, in order to use thermal effects to analyze and fully understand the mechanisms behind spin torque, soft magnetic materials with weak DW pinning are required so that thermal effects can be observed at room temperature without the application of external fields. Only then can very small currents sufficiently affect the magnetic system without significant heating, so that the size of the spin torque terms [Eq. (1)] can be determined using direct timeresolved high resolution imaging of the spin structure and comparison to a $1 \mathrm{D}$ analytical description of thermally induced DW dynamics [7,18]. However, for DWs in soft magnetic materials such as Permalloy, in which DWs are complicated 3D objects (see vortex DW spin structure [Fig. 1(d)] and transverse DW spin structure [Fig. 1(e)]), it is necessary to clarify if the description based on the quasiparticle model can be used to determine the nonadiabatic spin torque.

In this Letter, we investigate in real time thermally activated DWs jumping between two positions in Permalloy $\left(\mathrm{Ni}_{80} \mathrm{Fe}_{20}\right)$ nanowires using transmission electron microscopy. This motion occurs at room temperature
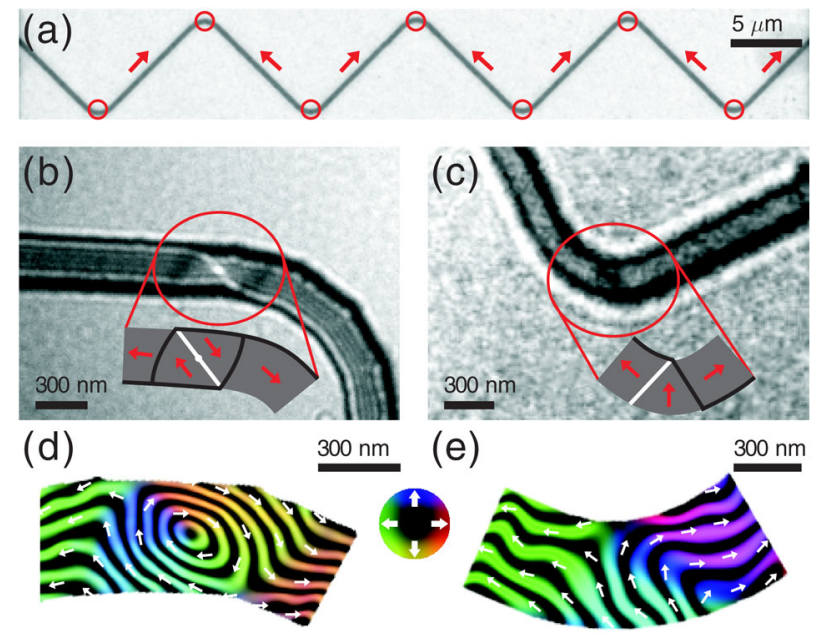

FIG. 1 (color online). (a) Bright field TEM image of a Permalloy zigzag line structure. The arrows indicate the directions of magnetization and the positions of nucleated DWs are marked by circles. (b) and (c) Fresnel images of thermally activated vortex and transverse DWs jumping between two pinning sites. (d) and (e) Electron holography magnetic induction maps of vortex and transverse DWs in the same wires showing detailed spin structures indicated by the color code and the arrows. in the absence of an external magnetic field or injected electron current and is of purely thermal origin. The distribution of the dwell times for which a DW stays at each of the two positions is well described by an Arrhenius law with the DW described as a quasiparticle in a 1D potential, with two metastable states separated by an effective energy barrier. By modifying the local potential using a small constant current with no significant heating and by analyzing the dwell times, we infer values for $\beta$ for a transverse and a vortex DW. We find that there are significant differences between the values determined for $\beta$ for different DW types, which can be attributed to their different spin structures.

Figure 1(a) shows a transmission electron microscopy image of a Permalloy zigzag wire on a silicon nitride membrane. These structures (wire width $150-500 \mathrm{~nm}$, wire thickness 8-20 nm) were fabricated by electronbeam lithography and a lift-off procedure [19]. At both ends, the line structures are contacted by Au pads, allowing for the injection of currents. To improve heat dissipation, the membrane substrates can be additionally back coated with $30 \mathrm{~nm}$ of aluminum.

Transmission electron microscopy is a particularly suitable technique to observe and understand the full thermally activated stochastic dynamics of DWs as it provides high spatial $(<10 \mathrm{~nm})$ and time resolution $(<0.05 \mathrm{~s})$ for single shot measurements. Here, the Fresnel mode of Lorentz microscopy is used to identify the positions of the DWs from the dark or bright contrast that results from the deflection of electrons by the Lorentz force [20]. For the investigation of detailed DW spin structures, we use offaxis electron holography [21].

The sample is initially magnetized using a field of $\sim 1 \mathrm{~T}$ in a direction perpendicular to the wires. Tilting the sample by $30^{\circ}$ results in the nucleation of DWs in the kinks of the wires after relaxing the field [see schematic in Fig. 1(a)]. We find either vortex [Figs. 1(b) and 1(d)] or transverse

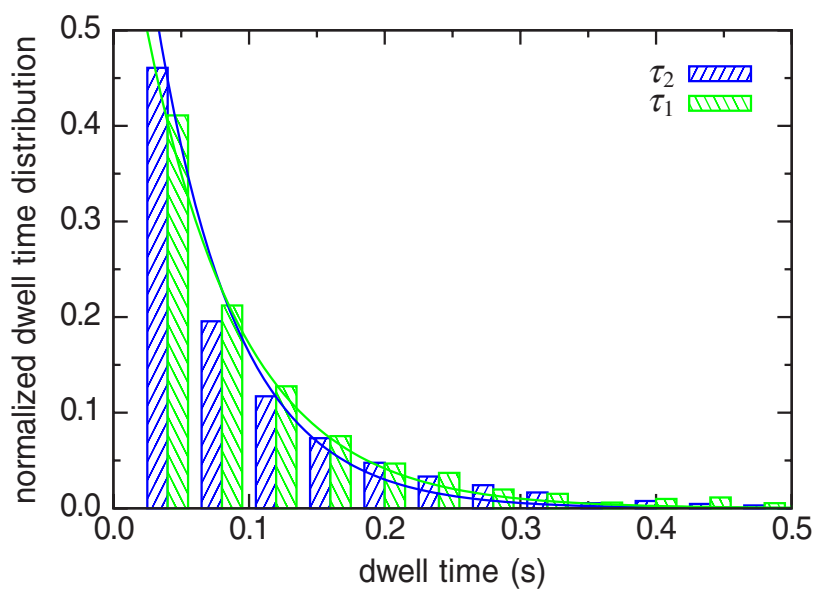

FIG. 2 (color online). Normalized distribution of dwell times at both pinning sites $\left(\tau_{1}\right.$ and $\left.\tau_{2}\right)$ of a thermally activated transverse DW in a Permalloy wire (sample 1) fitted with a single exponential function. 
[Figs. 1(c) and 1(e)] DWs depending on the wire geometry [15].

We first analyze a transverse DW (sample 1: wire width $495 \mathrm{~nm}$, wire thickness $10 \mathrm{~nm}$ ) jumping between two pinning sites $p_{1}$ and $p_{2}$ (separation $318 \pm 32 \mathrm{~nm}$ ) without the influence of an external magnetic field or current. This pure thermal DW motion occurs at room temperature since no significant heating effects due to the electron beam of the transmission electron microscope were observed (the sample temperature is monitored by measuring the resistance [22]). After acquiring 7500 Lorentz images of the DW (DW width $590 \pm 10 \mathrm{~nm}$ ) in the movie mode, we identify the DW positions and investigate the dwell times $\tau_{1}$ and $\tau_{2}$ of the DW at each of the two positions.

The exponential decay of the dwell time distributions (Fig. 2), and, in particular, the fact that it is well approximated by a single exponential function [23], means that the DW can be considered as a quasiparticle moving in a $1 \mathrm{D}$ potential between two metastable states separated by an effective energy barrier [shown schematically in Fig. 3(a)] [14]. In general, the pinning strength is not equal at the two positions, yielding two depths for the potential wells with two different energy barriers $\epsilon_{0,1}$ and $\epsilon_{0,2}$. The probability of a transition to the neighboring state is only determined by the ratio between the corresponding energy barrier and the thermal energy $k_{B} T$ that excites the transition. This dependence results in finite dwell times $\tau_{1}$ and $\tau_{2}$, for the DW at each of the two local metastable states.

We carry out the same investigation for a vortex DW (sample 2: wire width $395 \mathrm{~nm}$, wire thickness $17 \mathrm{~nm}$ ). The two pinning positions (separation $20 \pm 4 \mathrm{~nm}$ ) of the DW
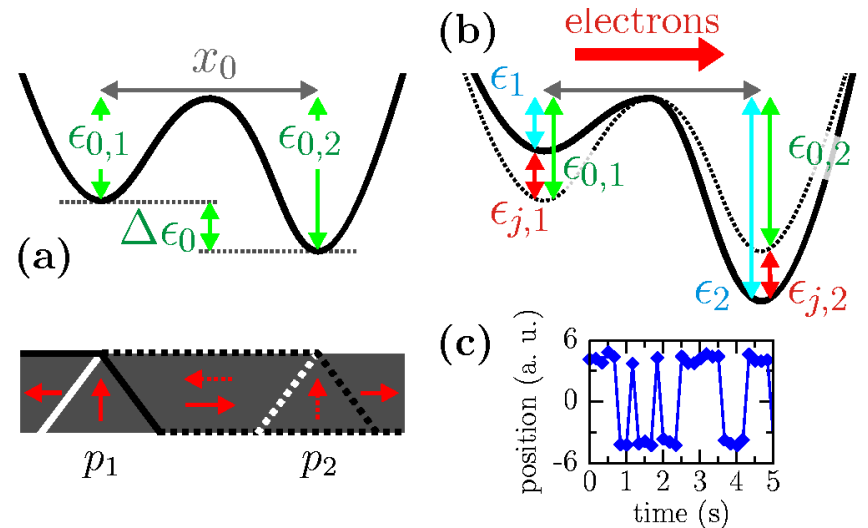

FIG. 3 (color online). (a) Double well potential for a 1D description of thermally activated DWs jumping between two positions $p_{1}$ and $p_{2}$ (schematically shown for a transverse DW). For the case without a current, the energy barriers consist of current independent contributions $\epsilon_{0,1}$ and $\epsilon_{0,2}$. The difference between the two barriers is $\Delta \epsilon_{0}$; the distance between the two pinning sites is $x_{0}$. (b) An additional force on the DW results from the injection of a spin-polarized current due to the nonadiabatic spin torque. The energy barriers now consist of current independent $\epsilon_{0, i}$ and current dependent contributions $\epsilon_{j, i}(i=1$, 2). (c) An example of measurements of the time-resolved thermal movement.
(DW width $363 \pm 10 \mathrm{~nm}$ ) are again extracted from Lorentz [Fig. 1(b)] and holography images [Fig. 1(d)] and we find that the complete DW is displaced. Again, the DW hops between the two pinning positions and the dwell times show the same exponential decay as the transverse DW, suggesting that the DW transitions can again be described by an Arrhenius law.

Since the dwell times of both DW types obey an Arrhenius law, we use in the following a 1D quasiparticle description [7] to analyze the influence of a constant direct current on the dynamics of the thermally activated DWs. In this model, the nonadiabatic torque acts as a force on the $\mathrm{DW}, F_{\mathrm{DW}}=-N \hbar \beta \tilde{u}_{x} / \lambda^{2}$ where $N$ is the number of spins in the DW, $\tilde{u}_{x}$ is an effective spin current drift velocity, and $\lambda$ is the DW width $[7,24,25]$. This force affects the 1D potential [Fig. 3(b)] and the current dependent shift in the energy $\epsilon_{j}$ is

$$
\epsilon_{j}=\frac{N \hbar \beta \tilde{u}_{x}}{\lambda^{2}} x=\frac{2 A \hbar \beta P}{e} \frac{j}{\lambda} x,
$$

with $N=2 \lambda A / a^{3}$ and $\tilde{u}_{x}=a^{3} P j / e, A$ the cross-sectional area of the DW, $a^{3}$ the volume of the unit cell, and the spin polarization $(P=0.37[26])$. The characteristic dwell times, for which the DW stays at each of the two positions are described by the Arrhenius law [4]:

$$
\frac{\tau_{1}}{\tau_{2}}=\frac{\tau_{0,1}}{\tau_{0,2}} e^{\left[\epsilon_{0,1}+\epsilon_{j, 1}-\left(\epsilon_{0,2}+\epsilon_{j, 2}\right)\right] /\left(k_{B} T\right)}
$$

Since the small current densities only induce a linear contribution to the current independent pinning potential [Eq. (2)], the curvature of the potential at the two metastable states, which is given by the second derivative, is not significantly affected by the applied currents. Thus, the attempt frequencies $1 / \tau_{0,1}$ and $1 / \tau_{0,2}$ determined by the potential curvature [4] are independent of the current. From Eqs. (2) and (3), we obtain

$$
\ln \frac{\tau_{1}}{\tau_{2}}=\ln \frac{\tau_{0,1}}{\tau_{0,2}}+\frac{\epsilon_{0,1}-\epsilon_{0,2}}{k_{B} T}+\frac{2 A \hbar \beta P}{k_{B} T e} \frac{x_{0}}{\lambda} j .
$$

By measuring dwell times as a function of current density and fitting these with Eq. (4), we obtain values for $\beta$.

For a transverse DW (sample 1), we measure the dependence of dwell times on the intensities and directions of
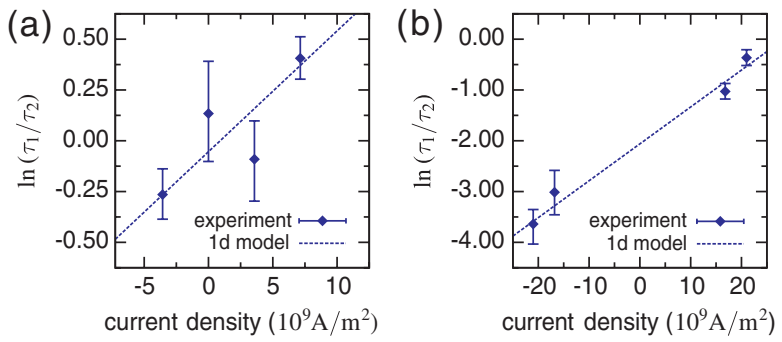

FIG. 4 (color online). Measurements of dwell times $\tau_{1}$ and $\tau_{2}$ plotted as $\ln \left(\tau_{1} / \tau_{2}\right)$ versus applied direct current for (a) transverse and (b) vortex DWs. The nonadiabatic coefficient $\beta$ is calculated from the slope of the fit with the 1D model [Eq. (4)]. 
constant direct currents. For each current density, 7500 Lorentz images are acquired in order to obtain sufficient statistics when extracting the positions of the DW [see example in Fig. 3(c)]. The resultant dwell times for both metastable states [Fig. 4(a)] are fitted to the 1D model [Eq. (4)] in order to calculate $\beta$ from the slope of the fit. From the Lorentz images and the electron holograms, values for the jump distance $x_{0}$ and the DW width $\lambda$ are derived, and from the fit in Fig. 4(a) we obtain $\beta_{t}=$ $0.010 \pm 0.004$ as the result. This value is similar to the value of the Gilbert damping constant measured for our Permalloy, $\alpha_{\text {Py }} \approx 0.008$ [27].

For the vortex DW (sample 2), positions and dwell times are again extracted from Lorentz images, as shown in Fig. 4(b), to obtain a value for $\beta_{v}=0.073 \pm 0.026$, which is considerably larger than the Gilbert damping constant. This measurement results in a larger nonadiabatic coefficient for the vortex DW than for the transverse DW $\left(\beta_{t} \approx\right.$ $1.3 \alpha_{\mathrm{Py}}$ for the transverse DW; $\beta_{v} \approx 9.2 \alpha_{\mathrm{Py}}$ for the vortex DW).

The fact that $\beta_{v} \gg \beta_{t}$ can be explained by using a more sophisticated description of the nonadiabatic torque, which includes two contributions $\beta_{v}=\beta^{n}+\beta^{s}$ with $\beta^{n}$ accounting for nonadiabatic transport and $\beta^{s}$ for spin relaxation due to spin-flip scattering [28]. Since the magnetization inside a transverse DW varies slowly, the corresponding magnetization gradients are small, and nonadiabatic contributions to the spin torque effect are mainly the result of spin-flip scattering due to impurities and spinorbit coupling [7]. Therefore, $\beta_{t} \approx \beta^{s}$ is expected for a transverse DW in Permalloy and values of the order of the damping constant $\alpha$ are predicted [12,29]. For a vortex DW in the investigated Permalloy nanowires, the magnetization turns out-of-plane at the vortex core and a large magnetization gradient occurs. Thus, nonadiabatic transport due to reflected conduction electrons becomes more significant and $\beta^{n}$ is larger [8], while the contribution due to spin relaxation $\beta^{s}$ is still present. Assuming that the contribution from spin relaxation $\beta^{s}$ is similar for transverse and vortex DWs as it is intrinsic to the material, we can derive the pure contribution of nonadiabatic transport $\beta^{n}=\beta_{v}-$ $\beta_{t}=0.063 \pm 0.030$. We note that these results are deduced from analysis based on a 1D model, which nevertheless seems to be a reasonable assumption given the good fit to the Arrhenius law in Fig. 2.

In conclusion, we derive $\beta$ from thermally activated vortex and transverse DWs obtaining $\beta_{v}=0.073 \pm 0.026 \gg$ $\beta_{t}=0.010 \pm 0.004$. The difference between these values is attributed to the high magnetization gradients at the vortex core. The comparatively large value for $\beta_{v}$ means that high DW velocities $(>100 \mathrm{~m} / \mathrm{s})$ should be possible for vortex walls, which bodes well for fast switching devices based on DW motion.

We acknowledge support by the German Science Foundation (DFG SFB 767, KL 1811), EU (RTN
Spinswitch MRTN-CT-2006-035327, Stg MASPIC ERC2007-Stg 208162, Stg NEWSPIN ERC-2007-Stg 201350), and the Samsung Advanced Institute of Technology. M.E. thanks the German National Academic Foundation.

*Also at Center for Electron Nanoscopy, Technical University of Denmark, 2800 Kongens Lyngby, Denmark.

${ }^{\dagger}$ Also at Laboratory for Micro- and Nanotechnology, Paul Scherrer Institut, 5232 Villigen PSI, Switzerland.

${ }^{\star}$ Also at Laboratory of Nanomagnetism and Spin Dynamics, Ecole Polytechnique Fédérale de Lausanne (EPFL), 1015 Lausanne, Switzerland; SwissFEL, Paul Scherrer Institut, 5232 Villigen PSI, Switzerland. mathias@klaeui.de

[1] A. Yamaguchi et al., Phys. Rev. Lett. 92, 077205 (2004).

[2] M. Kläui et al., Phys. Rev. Lett. 94, 106601 (2005).

[3] S. S. P. Parkin, M. Hayashi, and L. Thomas, Science 320, 190 (2008).

[4] P. Hänggi, P. Talkner, and M. Borkovec, Rev. Mod. Phys. 62, 251 (1990).

[5] A. Thiaville et al., Europhys. Lett. 69, 990 (2005).

[6] S. Zhang and Z. Li, Phys. Rev. Lett. 93, 127204 (2004).

[7] G. Tatara, H. Kohno, and J. Shibata, Phys. Rep. 468, 213 (2008).

[8] J. Xiao, A. Zangwill, and M. D. Stiles, Phys. Rev. B 73, 054428 (2006).

[9] C. Schieback et al., Eur. Phys. J. B 59, 429 (2007).

[10] M. D. Stiles et al., Phys. Rev. B 75, 214423 (2007).

[11] N. Smith, Phys. Rev. B 78, 216401 (2008).

[12] S. E. Barnes and S. Maekawa, Phys. Rev. Lett. 95, 107204 (2005).

[13] C. Schieback et al., Phys. Rev. B 80, 214403 (2009).

[14] C. Burrowes et al., Nature Phys. 6, 17 (2010).

[15] M. Kläui, J. Phys. Condens. Matter 20, 313001 (2008).

[16] M. T. Bryan, D. Atkinson, and R. P. Cowburn, Appl. Phys. Lett. 85, 3510 (2004).

[17] R. L. Compton, T. Y. Chen, and P. A. Crowell, Phys. Rev. B 81, 144412 (2010).

[18] M. E. Lucassen and R. A. Duine, Phys. Rev. B 80, 144421 (2009).

[19] D. Backes et al., Microelectron. Eng. 83, 1726 (2006).

[20] J. N. Chapman, Mater. Sci. Eng. B 3, 355 (1989).

[21] R.E. Dunin-Borkowski, M.R. McCartney, and D.J. Smith, Encyclopedia of Nanoscience and Nanotechnology (American Scientific Publishers, Stevenson Ranch, CA, 2004), Vol. 3, p. 41.

[22] M. Laufenberg et al., Phys. Rev. Lett. 97, 046602 (2006).

[23] J. P. Attané et al., Phys. Rev. Lett. 96, 147204 (2006).

[24] R. A. Duine, A. S. Núñez, and A. H. MacDonald, Phys. Rev. Lett. 98, 056605 (2007).

[25] M. E. Lucassen et al., Phys. Rev. B 79, 224411 (2009).

[26] R. J. Soulen et al., Science 282, 85 (1998).

[27] J. Walowski et al., Phys. Rev. Lett. 101, 237401 (2008).

[28] G. Tatara and P. Entel, Phys. Rev. B 78, 064429 (2008).

[29] Y. Tserkovnyak et al., Phys. Rev. B 74, 144405 (2006). 